\title{
Comparative Trial of an Alternative to the Bite Rim Record
}

\section{Mark Seiter}

Nature's Smiles, 575 East 4500 South B115, Salt Lake City, Utah 84107, United States.

info@naturessmiles.com

*Corresponding Author: Mark Seiter, Nature's Smiles, 575 East 4500 South B115, Salt Lake City, US.

\section{Abstract}

Background: The authors tested the hypotheses that the Staub Cranial System is an alternative to the traditional bite rim record.

Methods: A random sample of 19 edentulous patients who were rehabilitated by means of conventual complete dentures were now fitted with dentures fabricated using the Staub Cranial System. The participants completed an intake survey about their conventional denture. An identical survey about their Staub Cranial denture was taken after completion of the new prosthesis. Photographic records of the two dentures were evaluated by an independent blinded panel. Clinical notes for each participant were analyzedin this evaluation of the Staub Cranial technique.

Results: The patient's experience, the clinical evaluation and the photographic records all correlated positively in favor of the results achieved with the Staub Cranial System.

Practical Implications: The bite rim appointment can be eliminated. The system is sufficiently accurate to allow the bite to be registered at the try-in appointment.

Digital fabrication methods for full dentures are potentially enhanced by a system based in math and science. If the system can be digitized and enhanced with other digital markers, the denture workflows could go fully digital.

\section{The Missing Component}

Denture making is going digital. Labs are scanning impressions or models. Some dentists are using interoral scanners with the newest stitching technology to accurately capture full arch edentulous anatomy. Everimproving software enables quick and accurate designs. The new digital work flows bring cost saving efficiency that eliminates dental stone and wax. The one piece that remains subjective and somewhat arbitrary is where to place the teeth. Our digital protocols still require the base plate and bite rim or some variation that may involve a modified mush bite or gothic arch tracing or templates or impression tray techniques. All these are heavily dependent on the judgment and skill of the clinician and stand in stark contrast to the math and science-based techniques that characterize the rest of the digital denture process. To date, the digital process does not assist the dentist with the heavy burden of identifying overall vertical dimension, the occlusal plane, midline, incisal edge placement etc. Is there an alternative to the base plate and bite rim? Is there a way to determine tooth position that is based in math and science?

\section{THE SYSTEM}

In the year 2000, Karl Heinz Staub, creator of the Staub-Cranial Technology, asserted that for each individual there is one and only one ideal position for the teeth. That position would maximize esthetics, enable efficient and effective (masticatorial) function and allow for the optimal phonetic pronunciation of speech. Further, Mr. Staub boldly declared, "I know the position for the teeth." Such assertions are open to academic review and verification. In a doctorial study at the University of Freiberg, researchers found twenty candidates with healthy dentition and who had not received orthodontic treatment. (Figure 1) 


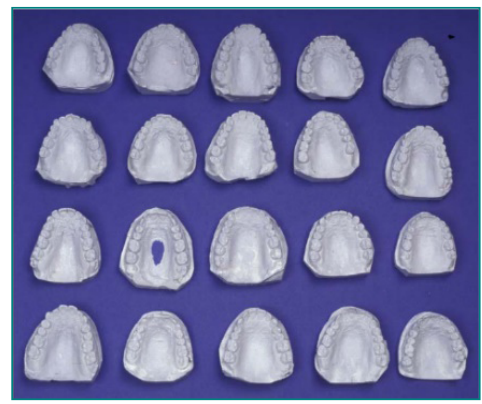

Fig 1

These individuals had teeth where mother-nature intended them to be. The casts were duplicated, and the teeth were removed from the set given to $\mathrm{Mr}$. Staub with the challenge to put the teeth back in their original position. (Figure 2)

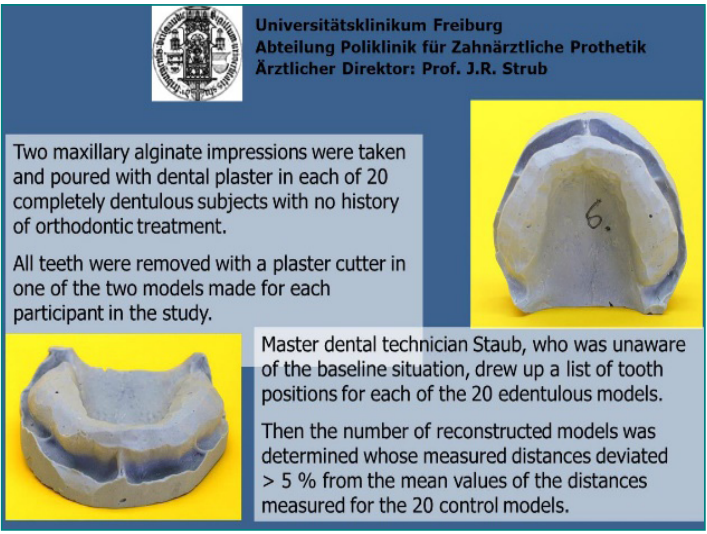

Fig 2

Tooth size and placement would be determined by the Staub Cranial System using denture teeth. When Mr. Staub was able to place the teeth on the casts with less than five percent deviation from the mean, the StaubCranial technique was recognized as a breakthrough. (Figure 3)

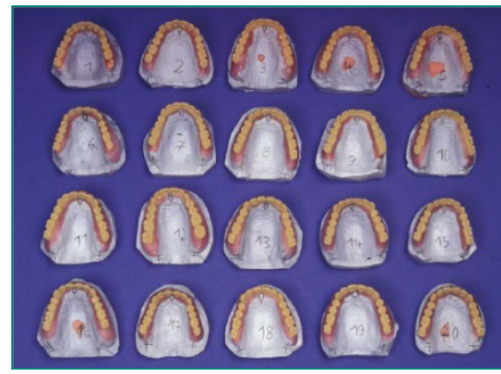

Fig 3

For the first time science and math was used to map the placement of the dentition and guide the setting of the teeth. The technology uses bone tissue reference points that can be identified on the master cast. These Cranial Reference Points are independent of the dental status; they are always present, always identifiable and always reproducible. Figures 4, 5 and 6

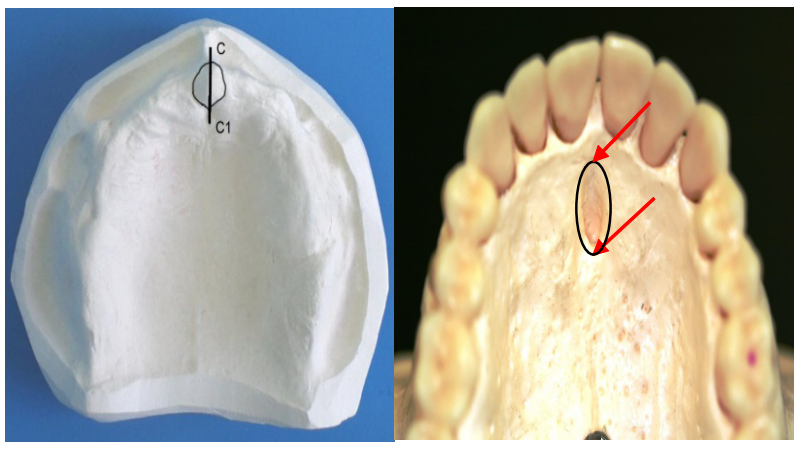

Fig 4

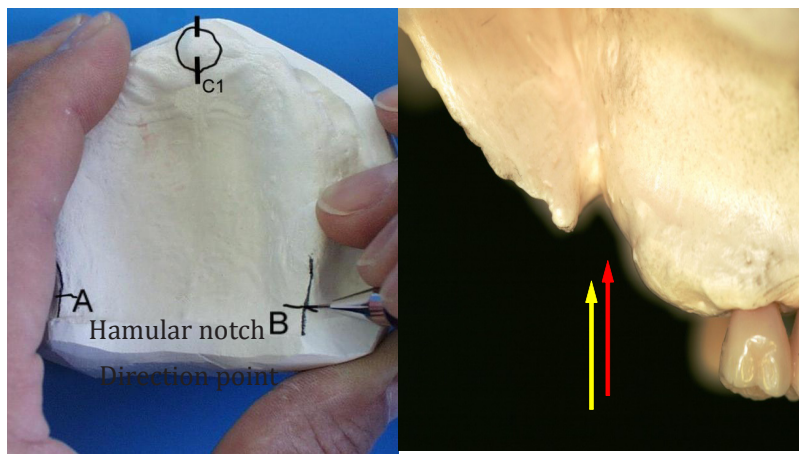

Fig 5

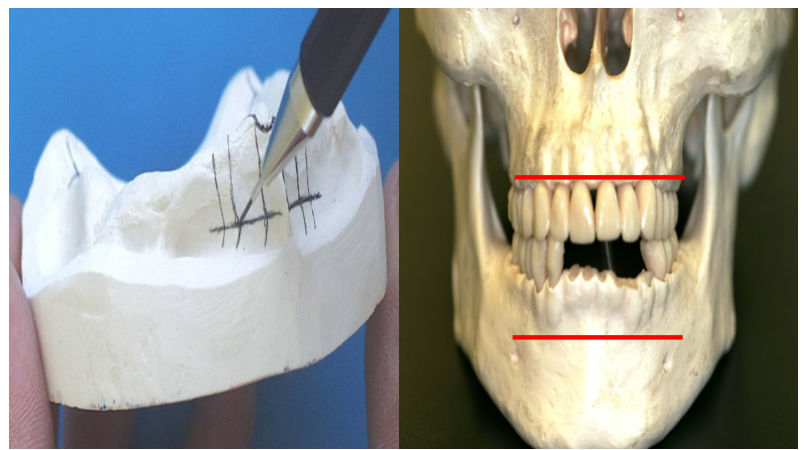

Fig 6

Illustrate the induction points, direction points and conclusion lines that provide the references for the computer program to mathematically generate the model map that definestooth position for the specific patient. These same points and the Staub Cranial devices allow the technician to tri-dimensionally align the model and mount it on the articulator without the use of a face bow transfer. With just two models and a shade, the laboratory can return the case with teeth set in wax for try-in and thus by-pass the bite rim appointment.

\section{THE STUdY}

After just such a brief explanation of the system, a prominent board-certified prosthodontist responded, "The solution is not found in a system. My belief is that the secret lies with the doctor. The understanding of the 
doctor serves the patient -- or not. The doctor sees the patient, prepares the patient, makes the impressions, determines the extension of the prostheses, specifies tooth position, midline, occlusal plane, placement relative to the joints, and OVD."(Dr. David Palmer,) We are in full agreement. The test of any system is if it can deliver results that meet the doctor's specification in all parameters. Dr. Mark L. Christensen, DDS, and Mark Seiter, CDT, have conducted this randomized trial to determine the effectiveness of the Staub Cranial System as an alternative for the bite rim record. This pragmatic study using unselected participants under flexible conditions includes randomly sequenced treatments comparing conventional dentures using the traditional bite rim with dentures fabricated using the Staub Cranial system. The hypothesis is that a set up that is guided by the system will eliminate the bite rim and its variations and will result in a denture that will equal or exceed the desired phonetics, function and esthetics of a conventionally fabricated denture. To test this hypothesis the study was designed to include input by the patient, an evaluation of clinical results and scores given by an independent panel from the photographic record comparing the conventional and Staub Cranial fabricated dentures.

Each of the nineteen patients in the sample has an existing functional, conventionally fabricated complete denture on one or both arches. On the intake survey they were asked about the comfort, appearance function and phonetic qualities of their current denture. Eighty-four percent were pleased with their current denture in one or more of these aspects. A series of photos were taken of the patient wearing the current conventionally made denture. Dr. Christensen completed a thorough inter oral examination upon intake. The study did not utilize Staub's condylar code technology for the analysis of joint dysfunction, and in fact, all candidates were generally healthy and free from any adverse oral conditions. The delivery of the Staub Cranial denture was completed in three to four appointments. At the first visit, alginate impressions were taken of both arches using a syringe to inject impression material onto the areas of the hamular notch and retromolar pads and into the vestibule. A loaded stock tray was placed using a static impression technique. The second appointment was for the wax try-in with teeth. For patients receiving a full upper and lower denture, the fourteen upper denture teeth were set to the Staub map. The six lower anteriors were also set up independent of the upper to the parameters specified by the system. A wax bite block was placed in the position of the lower posterior teeth. (See Figure 7)

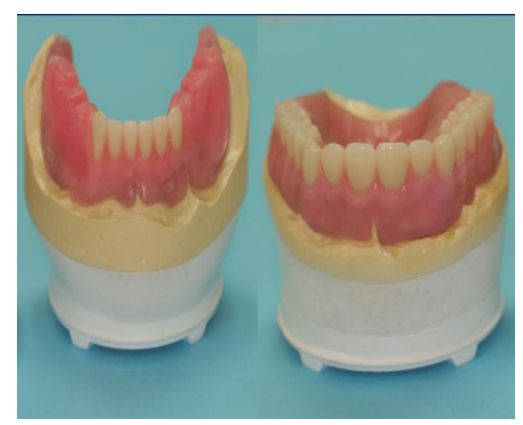

Fig 7

For patients with natural lower dentition, the upper six anterior teeth were set in wax with wax bite blocks in the posterior. This allows for the registration of the bite at the wax try-in. The bite blocks are made to the dimensions specified by the system. In all but one of the nineteen cases the occlusal plane was accurate to within .5 to $1.5 \mathrm{~mm}$. Few adjustments weremade to the wax rims. The tooth placement was evaluated. A bite registration material was extruded and the relationship was captured. An optional appointment was offered for those participants who wanted to see the final wax-up before processing. A compilation of the changes and clinical notes from this extra appointment are a key part of the findings of this study. The final appointment was the delivery of the Staub Cranial Prosthesis. It was not possible to blind the investigators or the participants. The evaluation of the data, however, was blinded and care has been taken to achieve a statistically significant result. The body of data gathered from the study is presented in three parts. An evaluation of the photographic record, analysis of the clinical notes and tabulation of the patient survey follow.

\section{Evaluation of The Photographic Record}

This superiority trial was judged by a panel of seventeen general dentists evaluating sets of four photos of patients wearing the conventionally or Staub Cranial made denture. They were asked to score the photos based on appearance. In their professional opinion does the denture appear to support and enhance the patient's appearance. Without knowing what denture they were evaluating, they were asked to give each photo a rating--favorable plus, unfavorable minus, or no opinion, zero. This was followed by a four photo 
side-by-side evaluation. Figure 8 is an example of the side-by-side photos. The doctors gave the photos with the conventionally fabricated dentures an average of 6.7positive scores. They gave the photos with the Staub Cranial dentures an average of 11.5 positive scores. When the photos of the conventionally fabricated and Staub Cranially fabricated denture were displayed side-by-side, the conventionally fabricated dentures received an average of 3.7 positive scores. They gave the photos with the Staub Cranial dentures an average of 10.6 positive scores.

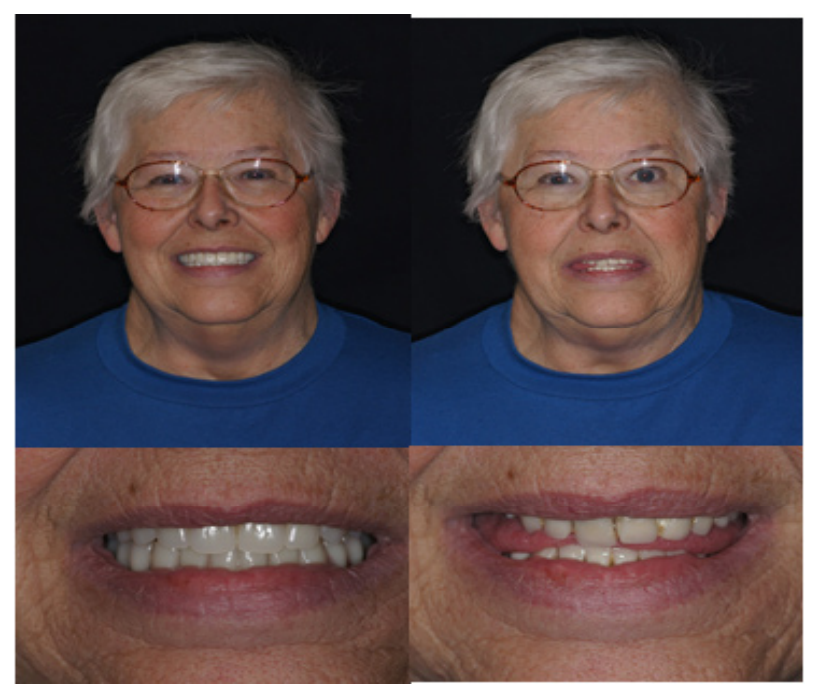

\#19X

\#19Y

Fig 8

\section{Analysis of the Clinical Notes}

Restoration, by definition, is to bring back that which was lost. The new prosthesis should reestablish the dental status that existed before the degradation and loss of their natural teeth, assuming an ideal physiology originally existed. At the try-in appointment, study participants were told, "This is where the computer suggests your own teeth were originally, what do you think?" They were encouraged to express their true feelings and were guided in their critique as they would focus first on midline accuracy, then on incisal placement and labial position. Questions were asked such as, "Do your teeth appear to be level?" and "Does your lip look full enough?” etc.

Twenty-one percent made no changes to the Staub Cranial set-up. Ten percent requested one change, Forty-two percent wanted two changes, twenty-one percent asked for three changes and five percent four changes. In the Staub Cranial system, the upper and lower dentures are set independent of one another to a computer generated map. The first time the two setups articulate is when they are placed in the mouth and the patient is asked to close. If a patient was receiving a full upper and lower denture and the patient wanted the midline changed, changing the upper and lower midline was counted as two changes.

An important finding was the degree of change that was requested. The nineteen participants requested a total of 34 changes. Of those changes, fifty-six percent were changes of $1 \mathrm{~mm}$ or less. Thirty-five percent were changes of 1.5 to $2 \mathrm{~mm}$. Six percent were changes of 2.5 to $3 \mathrm{~mm}$ and there was one change of $7 \mathrm{~mm}$. Changes to the mid-line and to the incisal plane were equally frequent with fewer changes made to labial placement.

The system claims to be capable of delivering an accurately fabricated denture from impressions to delivery in three appointments. Thirteen of the fifteen study participants who requested changes returned for an optional second try-in appointmentto verify and approve those changes. Four participants wanted further changes. These patients had requested three or more changes to the Staub Cranial try-in and had very high expectations. $79 \%$ of the participants made no changes to the try-in or approved adjustments without further change. For these patients, three appointments are sufficient.

In every case, post-delivery appointments were to address sore spots. Seventy-five percent of participants were free of any discomfort in two or fewer follow-up visits. Twenty-five Percent required three or more visits.

\section{Compilation of the Patient SuRvey}

The restorative process is or should be about the patient. Patient health, functional ability and satisfaction are primary measures of our success. A survey was taken as the patient entered the study with questions that focused on their current conventionally made denture. They were asked if they were pleased with their denture's comfort, appearance, function and phonetic qualities. The survey also included questions about the ease of the appointments, how well they were listened to, how closely the denture duplicated their natural teeth and their overall experience in having the denture made. An identical survey was taken at the end of the study asking the same questions about their new Staub Cranial made denture. $35 \%$ were pleased with the comfort of their 
conventional denture and $80 \%$ with the Staub denture.

$65 \%$ were pleased with the conventional denture and $95 \%$ with the Staub denture. $40 \%$ were pleased with the function of their conventional denture and $75 \%$ with their Staub denture. $60 \%$ were pleased with the phonetics of their conventional denture and $75 \%$ with their Staub denture. When asked to rate their experience on a scale of one to five with one as the most positive, the Staub Cranial experience received a 1.6. Their experience being fitted with the conventional denture was a middle of the road 2.4.

\section{STUDY WEAKNESSES}

While it is true that the Staub Cranial System extracts important patient specific information from the oral anatomy to more quickly and accurately position the teeth, the investigator acknowledges that the stronger positive patient experience cannot be completely attributed to the system. The attentive care and professional treatment from Dr. Mark L. Christensen and his staff made a strong impression with all the study participants. The investigator also gratefully acknowledges Ivoclar North America for contributing the Phonares NHC teeth that were used in the dentures. The conventionally made denture may not have had the advantage of such a quality product to enhance the denture's appearance.

\section{ConClusions}

Based on appearance, the Staub Cranial denture was preferred over the conventionally made denture. The panel of dentists gave photos of the Staub dentures more than twice the favorable scores than were given the conventional dentures.

The study showed that the Staub denture simplifies and shortens the clinical experience without a compromise in quality. $80 \%$ of dentures can be successfully delivered in three appointments. 20\% of patients--those with excessively high expectations and requesting multiple changes needed extra appointments. Over ninety percent of the changes were changes of $2 \mathrm{~mm}$ or less. These were often a reflection of personal preference in a dynamic exchange between the patient and the clinician. At the second appointment the patient was engaged in the decision making process--a process that allowed them to take ownership. When asked to rate their clinical experience, the patients said that the Staub Cranial method was easier, provided opportunity for their input, resulted in a denture similar to their natural teeth and presented fewer challenges for adjusting to the new denture.

The patients rated the Staub Cranial denture as being more comfortable, more functional, and easier to speak with and better looking than their conventional denture.

Participation in the study was free of charge. However, the investigator wished to know if the patient would be willing to pay for the new denture. In the intake interview the participant was told that $\$ 180$ per denture was required if they wanted to keep the denture. This amount was high enough that if the patient truly felt that their conventional denture was superior, they would be unwilling to spend the money. If the Staub Cranial denture were superior, the amount would not be so high that cost would influence the decision. One denture was returned at the end of the study. It was the denture shown in the side-by side photos in Figure 8.

"...the secret lies with the doctor. The understanding of the doctor serves the patient -- or not. The doctor sees the patient, prepares the patient, makes the impressions, determines the extension of the prostheses, specifies tooth position, midline, occlusal plane, placement relative to the joints, and OVD." Dr. David Palmer has accurately described where the responsibility rests. This burden and the probability for error have driven many clinicians away from what is perceived to be a difficult and unprofitable part of dentistry. This comparative trial of the Staub Cranial Technique clearly demonstrates that the system can successfully bypass the bite rim appointment making the doctor's role one of verifying all the parameters mentioned and prescribing small corrections as needed. The investigator is unaware of any other system that is based in math and science with the potential to digitize the one remaining traditional protocol - tooth placement. It is a firm hope that the digital workflow 
Comparative Trial of an Alternative to the Bite Rim Record

will simplify the doctor's role,increase the likelihood

for profitability and improve the environment for a rewarding full-mouth reconstruction experience for the doctor and the edentulous patient.

\section{REFERENCES}

[1] ZA PanosLampropoulos, Dipl.-Math. Dr. Thomas Gerds, PD Dr. Jens C. Turp. Reconstruction of tooth positions using the Staub Cranial System. Freiburg University Hospital, Germany.
Outpatient Clinic for Dental Prosthetics

[2] Pieter Prinsloo - Medunsa Oral Health Center. What is new in the quest for accurate Jaw Registrations. Southern Africian Dental Technology Journal, SADTJ, Vol 3 Issue 1, Feature Article

[3] Karl Heinz Staub. Staub Cranial, Healthy Dentures, Theoretical Summary. www.staub-cranial.de

Citation: Mark Seiter. Comparative Trial of an Alternative to the Bite Rim Record. Archives of Dentistry and Oral Health. 2019; 2(2): 18-23.

Copyright: (C) 2019 Mark Seiter. This is an open access article distributed under the Creative Commons Attribution License, which permits unrestricted use, distribution, and reproduction in any medium, provided the original work is properly cited. 